\title{
WAVE-RELATED SUSPENDED SAND TRANSPORT IN RIPPLE REGIME
}

\author{
Dang Huu Chung ${ }^{1}$, B.T.Grasmeijer ${ }^{2}$ and Leo C Van Rijn ${ }^{3}$
}

\begin{abstract}
This paper presents results of an experimental and theoretical study of wave-related suspended sand transport under irregular waves in the ripple regime. Time series analysis of experimental data to determine the mean and oscillating suspended transport components was done by using a specially developed software. The relationship between the wave-related suspended sediment transport rate and the significant wave height is established. The influence of particle diameter on the wave-related transport is also seen clearly through the analysis results. The second part of the study is related to the 1DV model, which simulates the instantaneous velocity and sand concentrations from close to the bed up to higher in the water column. The model is based on the classical diffusion approach taking both the turbulence-related and the effective wave-related diffusion into account. It shows that the time-averaged sand concentrations can be simulated reasonably well in the ripple regime using the calibrated equations. The accuracy of the suspended transport was found to be strongly dependent on the type of applied bed-boundary condition for the sand concentration (computed from a formula or taken from measured data).
\end{abstract}

\section{Introduction}

The suspended sand transport rate in the coastal zone comprises two major components: the current-related transport component and the wave-related transport component. The current-related transport component is considered to be the convective sand transport carried by the mean currents such as tide, wind and wavedriven currents in the presence of short (high-frequency) surface waves acting as stirring agents. The wave-related sand transport is herein defined as the transport of sand particles by the oscillating (orbital) fluid motion due to the high-frequency waves. The current-related transport over rippled beds has been studied in considerable detail (Van Rijn et al., 1993; Van Rijn and Havinga, 1995), but the

\footnotetext{
${ }^{1}$ Institute of Mechanics, 224 Doi Can, Hanoi, Vietnam

Fax: 84-4-8333039, Email: Dhchung@im01.ac.vn

${ }^{2}$ Inst. for Marine and Atmospheric Res. Utrecht, Utrecht University

P.O. Box 80115, 3508 TC Utrecht, The Netherlands, Email: B.Grasmeijer@geog.uu.nl

${ }^{3}$ Delft Hydraulics, P.O.Box 177, 2600 MH, Delft, The Netherlands; Leo.vanrijn@wldelft.nl
} 
wave-related transport is less well known. Both the magnitude and direction of the wave-related transport vary depending on the precise geometry of the ripples: relatively flat ripples may result in onshore-directed transport, whereas steep vortexinduced ripples may yield offshore-directed transport due to phase differences between instantaneous fluid velocities and sand concentrations near the ripples (Vincent and Green, 1990; Osborne and Greenwood, 1992; Osborne and Vincent, 1996; Grasmeijer and Van Rijn, 1999, Van Rijn, 1993 and Van Rijn, 1998). The wave-related transport over a flat bed has been studied in more detail based on numerous experiments in wave tunnels (see for overview: Ribberink, 1998). Generally, the wave-related transport over a flat bed is found to be onshore-directed for sand particles larger than about $0.15 \mathrm{~mm}$, because phase differences are less important in the thin sheet flow layer.

This paper focuses on the wave-related suspended transport over a rippled bed based on results from both experimental and mathematical studies. The experimental study is related to large-scale wave flume tests and analysis of time series of horizontal velocity and suspended sediment concentration in the Delta Flume of Delft Hydraulics. The mathematical study is related to numerical model computations focussing on suspended sediment concentrations under irregular waves in the ripple regime based on a 1DV point-model. The present model is a modified version of the model of Ribberink and Al Salem $(1991,1995)$ and will herein be used to study the wave-related suspended sand transport in the ripple regime. Suspended sand transport for regular asymmetric waves was studied already in Ribberink and Al-Salem (1991) and in Ribberink and Al-Salem (1995) for sheet flow over a plane bed in a wave tunnel. They have found that the 1DV model produces realistic estimates of the sand concentrations and transport rates in the thin sheet flow layer over a plane bed using an effective bed roughness of $\mathrm{k}_{\mathrm{s}}=2.5 \mathrm{D}_{50}$. However, the time-averaged sand concentrations above the sheet flow layer are considerably overpredicted by the model, which implies that the turbulence-related mixing coefficient is overpredicted by the model in case of a plane bed. This effect was supposed to be related to the damping of turbulence caused by high concentrations in the sheet flow layer, which was not modelled.

In the present paper the attention is focussed on the performance of the $1 \mathrm{DV}$ model for irregular waves in the ripple regime. The results will be used to show whether a diffusion-type model can be applied to simulate the suspension mechanism by rippleinduced vortices. The vortex-induced transport process may well be a convective process related to the migrational behaviour of oscillating coherent ripple-induced vortices rather than a turbulence-induced diffusion process as pointed out by Nielsen (1992).

\section{Description of experiment}

The experiments were carried out in a large-scale wave flume with a total length of 233 meters, a depth of 7 meters and a width of 5 meters and waves were generated by a piston-activated board at the end of the flume. A sand bed layer was placed in the wave flume from position $\mathrm{x}=100$ meters to $\mathrm{x}=140$ meters. The water depth was 4.5 $\mathrm{m}$ in all experiments. Two types of sand have been used: fine sand with median diameter of $0.16 \mathrm{~mm}$ and coarse sand with median diameter of $0.33 \mathrm{~mm}$. Three different wave conditions for both regular and irregular waves were generated during the test series. In this paper the tests with irregular waves are considered only. The significant wave heights varied in the range of $H_{\mathrm{s}}=1$ to $1.5 \mathrm{~m}$. The peak wave period 
was $5 \mathrm{~s}$. The measurement program is described in Table 1, in which the tests with the same wave condition and the same grain size are arranged in five different sets (I,II,II,IV and V). The data sets I and II involve the tests with relatively coarse sand with a median diameter $\mathrm{d}_{50}$ of $0.33 \mathrm{~mm}$. The other sets (III, IV and V) deal with fine sand with $\mathrm{d}_{50}$ of $0.16 \mathrm{~mm}$.

\section{Analysis and results}

\subsection{Definitions and analysis method}

The instantaneous velocity and sediment concentration at height $\mathrm{z}$ above the bed are separated into time-averaged, high frequency and low frequency components. The net total transport rates at height $\mathrm{z}$ in cross-shore direction (positive direction of $\mathrm{x}$ axis is in wave propagation direction) are defined as the integration over the measurement time interval of instantaneous sediment transport rate, as follows

$$
\overline{u c}=\overline{u c}+\overline{\widetilde{U}_{s} \widetilde{C}_{s}}+\overline{\widetilde{U}_{s} \widetilde{C}_{l}}+\overline{\widetilde{U}_{l} \widetilde{C}_{s}}+\overline{\widetilde{U}_{l} \widetilde{C}_{l}}
$$

in which the following conventions are used:

$Q_{x}=\overline{u c} \quad$ : net cross-shore sediment transport rate

$Q_{a x}=\bar{u} \bar{c} \quad$ :time-averaged (current-related) cross-shore sediment transport

$Q_{s s x}=\widetilde{\widetilde{U}_{s} \widetilde{C}_{s}}:$ high frequency (wave-related) cross-shore sediment transport

$Q_{s l x}=\widetilde{\widetilde{U}_{s} \widetilde{C}_{l}} \quad$ : interaction between high and low frequencies

$Q_{l s x}=\widetilde{\widetilde{U}_{l} \widetilde{C}_{s}}:$ interaction between high and low frequencies

$Q_{l l x}=\overline{\widetilde{U}_{l} \widetilde{C}_{l}}:$ low frequency cross-shore sediment transport rate

“" $\quad$ : denotes time-integrated quantity

The analysis results of the experiments are obtained by using TISERAT(a software coded with FORTRAN 90) by which filtering of components of high and low frequencies and computation of the cross-shore transport rates were done.

Table 1. Measurement program

\begin{tabular}{clcccc}
\hline $\begin{array}{c}\text { Data } \\
\text { Set }\end{array}$ & Measurement No. & $\begin{array}{c}\text { Number } \\
\text { of test }\end{array}$ & $\begin{array}{c}\text { Grain size } \\
\mathrm{d}_{50} \\
(\mathrm{~mm})\end{array}$ & $\begin{array}{c}\text { Wave } \\
\text { height } \\
\mathrm{H}_{\mathrm{s}} \\
(\mathrm{m})\end{array}$ & $\begin{array}{c}\text { Orientati- } \\
\text { on of } \\
\text { ASTM } \\
(\text { degree })\end{array}$ \\
\hline I & $\begin{array}{l}\text { A1,A1B,A2A, } \\
\text { D1,D2,D3,D4,D5 }\end{array}$ & 8 & 0.33 & 1.00 & -90 \\
& & & & & -120 \\
II $\quad \begin{array}{l}\text { B1A,B2A,B1B, } \\
\text { E1,E2,E3,E4,E5 }\end{array}$ & 8 & 0.33 & 1.25 & -90 \\
III & $\begin{array}{l}\text { G1,G2,G3,G4, } \\
\text { J1,J2,J3 }\end{array}$ & 7 & 0.16 & 1.00 & -120 \\
IV & $\begin{array}{l}\text { H1,H2,H3,H4,H5, } \\
\text { K1,K2,K3,K4 }\end{array}$ & 9 & 0.16 & 1.25 & -120 \\
V & M1,M2,M4 & 3 & 0.16 & 1.50 & -90 \\
\hline
\end{tabular}




\subsection{Suspended sediment transport components}

Analysis of the results for all tests shows that the suspended sediment transport components due to interaction between high and low frequencies are very small and can be ignored, while the components due to purely high and low frequencies are relatively large. Hence, the net cross-shore suspended sediment transport now becomes the sum of time-averaged component and the components of high and low frequency transport:

$$
Q_{x}=Q_{a x}+Q_{s s x}+Q_{l l x}
$$

The terms of equation (2) are presented in Fig.1 showing the vertical distribution of suspended sediment transport components for each data set I to V (see Table 1).

In general, from the observation on Fig. 1 it can be seen that the transports due to high frequency are dominant and tend to be directed onshore. The transports due to low frequency are of minor importance and have a tendency for offshore direction similar to time-averaged transport components. It can be clearly observed that the suspended sediment transport mainly occurs in the near-bed layer with thickness of about 0.3 to $0.5 \mathrm{~m}$, which is roughly equivalent to 10 to 20 times the ripple height.

In order to provide an evaluation of depth-integrated transport rates, the transport terms have been integrated between the lowest and highest measurement points. In Figure 2 the time-averaged ( $Q_{\text {axd }}$, current-related) and the high-frequency ( $Q_{s s x d}$, wave-related) suspended transport rates are shown as a function of wave height and sediment size. In all conditions with irregular waves the wave-related suspended transport is directed onshore, whereas the current-related suspended transport is directed offshore. From Figure 2 it is easy to see that the wave-related suspended transport increases with wave height and decreases with particle size. This latter effect can be understood from the ripple dimensions; the ripples generated over the $0.33 \mathrm{~mm}$
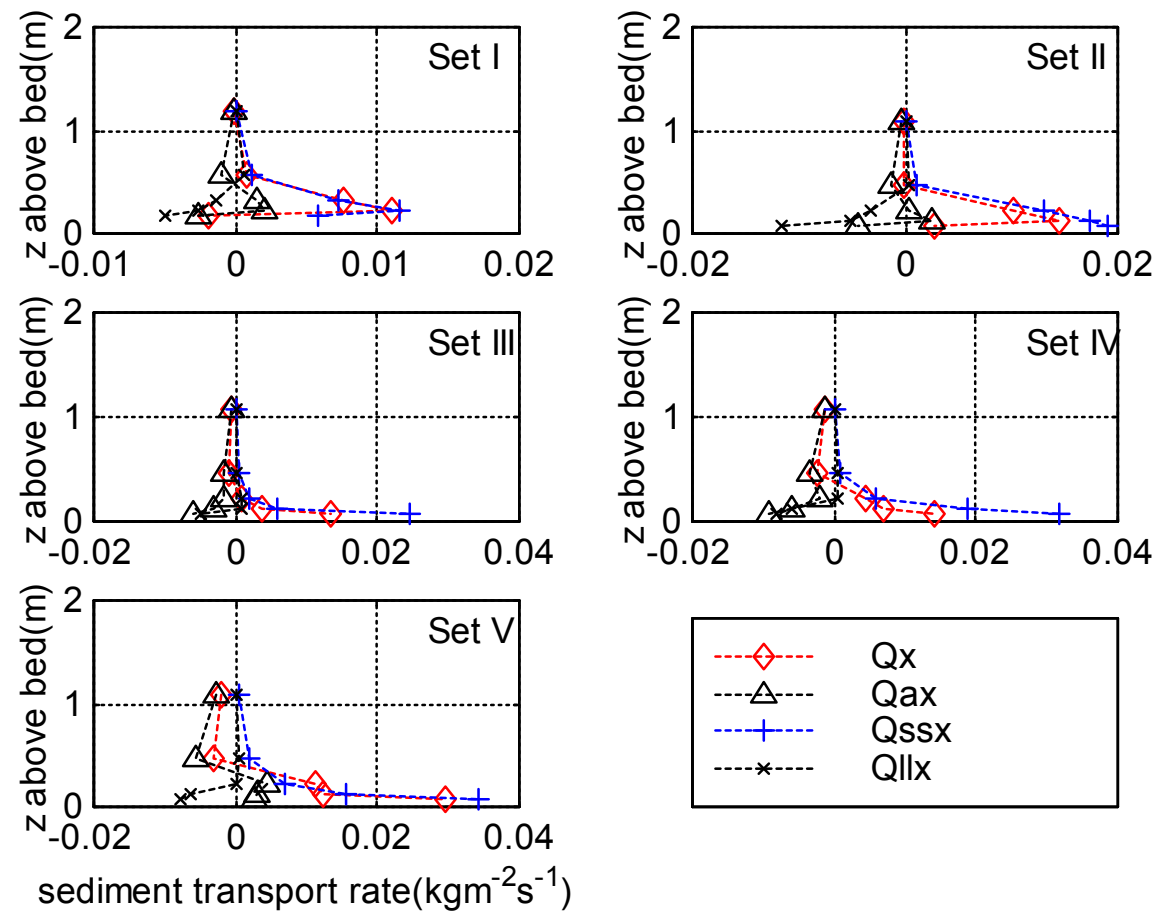

Fig. 1 Distribution of cross-shore sediment transport components for combined tests 
sand bed were much more pronounced as those for the $0.16 \mathrm{~mm}$ sand bed resulting in larger vortex motions and stronger associated suspension processes. The standard error of the wave-related transport is relatively large for the case with sand bed of $0.16 \mathrm{~mm}$ and significant wave height of $1.5 \mathrm{~m}$, expressing relatively large variability because only 3 data records of 15 minutes were available (see Table 1, data set V). It stresses the importance of relatively long data sets in case of a rippled bed.

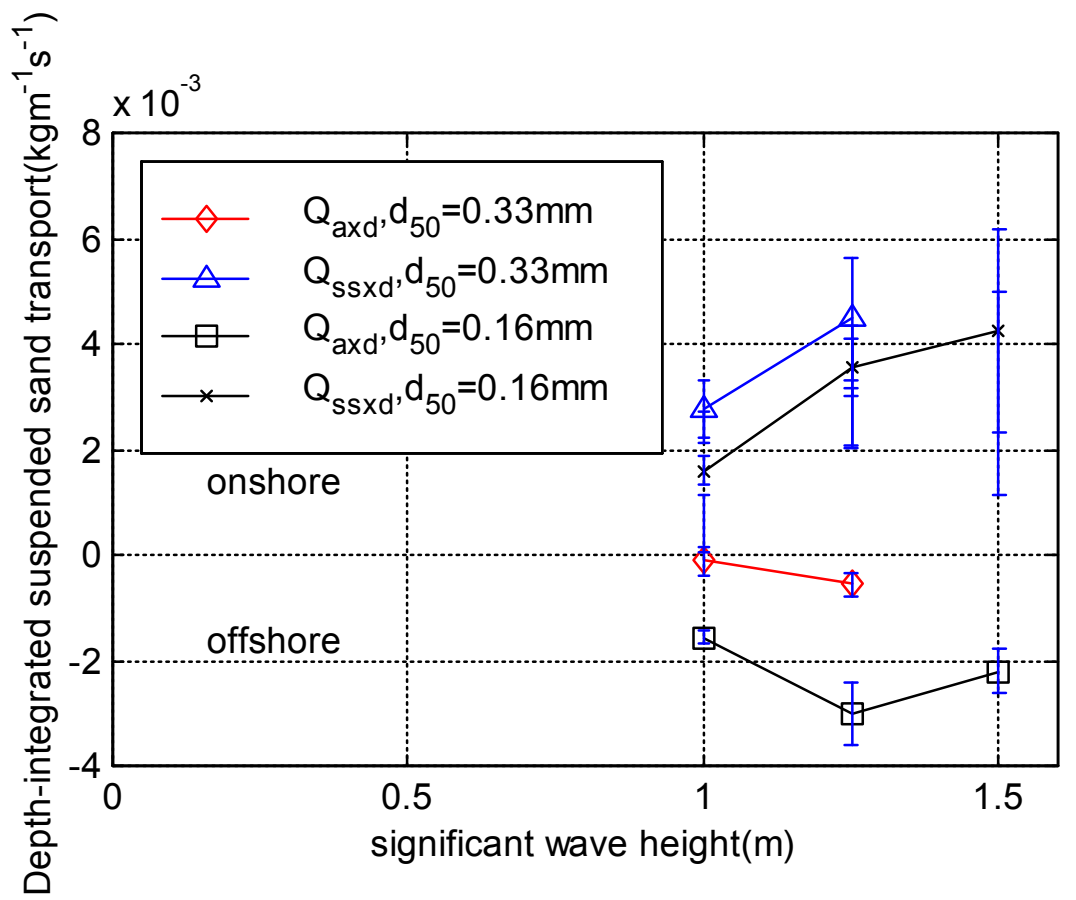

Fig. 2 Relation between depth-integrated transport, significant wave height and sediment size

\section{Mathematical model}

The model is a modified version (including irregular waves and vortex-related mixing) of the model explained by Ribberink and Al-Salem (1991 and 1995). Similar models have been used by other researchers such as Jonsson (1963), Kajiura (1968), Horikawa and Watanabe (1968), Bakker (1974), Brevik (1981), Fredsoe et al.(1985) and Rakha et al. (1997). Most often these models have been used for plane bed conditions, but Rakha et al. (1997) applied their model to compute the profile evolution in the surf zone with ripples along a considerable portion of the profile.

The turbulent fluid movement for (horizontal) uniform flow in the vertical plane is considered assuming that the vertical velocity is relatively small compared to the horizontal velocity. Furthermore it is assumed that the region of flow can be divided in two layers, in which the upper layer has no vertical gradient velocity. Hence, by using the term of pressure gradient from the equation for the upper layer, the movement in the lower layer is described by the Reynolds' equation as follows:

$$
\frac{\partial u}{\partial t}=\frac{\partial u_{0}}{\partial t}+\frac{\partial}{\partial z}\left(v_{t} \frac{\partial u}{\partial z}\right), z_{0} \leq z \leq h
$$


in which $u(z, t)$ is the horizontal velocity in the oscillatory lower layer with the depth $h, u_{0}$ the horizontal velocity at elevation $z=h$ distinguishing between the lower and the upper layer, $t$ time, $z$ elevation above bed, $v_{t}$ turbulent viscosity and $z_{0}$ position of zero velocity.

Eq.(3) is suitable to simulate the orbital flow field over a plane bed or a bed with relatively flat ripples (no flow separation). It can not simulate the detailed vortex motions over pronounced ripples, which basically is a horizontally non-uniform process. Since the attention is focussed on the wave-related suspended transport, eq.(3) is herein used to describe the general orbital motion and the turbulence-related mixing characteristics. The basic idea is to evaluate the performance of a relatively simple model with respect to the suspension characteristics over a rippled bed and to see whether the model can be adjusted to produce the proper wave-related transport rates or not.

In order to close the equation, the Prandtl's theory on mixing length as turbulence closure is used and the eddy viscosity is described by

$$
v_{t}=\beta_{1} l^{2}\left|\frac{\partial u}{\partial z}\right|
$$

in which $\beta_{1}$ is the eddy viscosity adjusting coefficient (equal to 1 ), $l$ is the mixing length and determined by Prandtl's formula:

$$
l=\kappa z
$$

where $\kappa$ is Von Karman constant $(\kappa=0.4)$.

The initial and boundary conditions used in the model are given in the following.

- The boundary conditions at $\mathrm{z}=\mathrm{h}$ and $\mathrm{z}=0$ :

$$
\begin{aligned}
& \left.u(z, t)\right|_{z=h}=f_{1}(t) \\
& \left.u(z, t)\right|_{z=z_{0}}=0
\end{aligned}
$$

in which $f_{1}(t)$ is a given function from measurement (data record of irregular wave velocities);

- The initial condition:

$$
\left.u(z, t)\right|_{t=0}=f_{2}(z), \quad z_{0} \leq z \leq h
$$

in which $f_{2}(z)$ is given function of velocity. A hyperbolic tangent distribution is used for the initial velocity based on the initial value at $z=h$.

Using the same assumptions as for flow equation (3), the vertical distribution of sediment concentration is described by the $1 \mathrm{DV}$ diffusion equation as follows:

$$
\frac{\partial c}{\partial t}+\frac{\partial}{\partial z}\left(w-w_{s}\right) c=\frac{\partial}{\partial z}\left(\varepsilon_{s} \frac{\partial c}{\partial z}\right), z_{a} \leq z \leq h
$$


in which: $w$ is the vertical velocity of flow, $w_{s}$ constant settling velocity of sediment particle, $c$ volume concentration, and $\varepsilon_{s}$ diffusion coefficient for sediment.

To represent additional vortex-related suspension processes, the overall sediment mixing coefficient was modelled as the sum of the ones by bottom-induced turbulence and effective vortex-induced mixing:

$$
\varepsilon_{s}=\sqrt{\varepsilon_{s t}^{2}+\varepsilon_{s w}^{2}}
$$

in which $\varepsilon_{s t}, \varepsilon_{s w}$ are the mixing coefficients for sediment due to turbulence and due to vortex motions, respectively. The turbulence-related sediment mixing coefficient is determined according to eddy viscosity $v_{t}$ by a coefficient $\beta_{2}$

$$
\varepsilon_{s t}=\beta_{2} v_{t}
$$

The vortex-induced mixing is supposed to be described by the following formula:

$$
\varepsilon_{s w}=\frac{1}{n} \hat{U} z\left(1-\frac{z}{h}\right)
$$

Eq.(12) is based on dimensional analysis and assuming that the sand concentration profile is of the hyperbolic type, $c \sim(h / z-1)^{n}$, with $\mathrm{n}$ being a calibration coefficient most likely depending on grain size and ripple dimensions. Furthermore, it is assumed that peak orbital velocities, $\hat{U}$, are dominant in driving the vortices. The peak orbital velocity is described as:

$$
\hat{U}=\frac{1}{2}\left(U_{o n}(z, T)+U_{o f f}(z, T)\right)_{z=h}
$$

in which $U_{o n}, U_{o f f}$ are onshore and offshore amplitudes of peak orbital velocity in case of irregular waves, respectively; $T$ wave period. The effective vortex-induced mixing has been determined by calibration using the measured time-averaged sand concentrations.

The boundary equations for the sediment diffusion equation are:

- The initial condition:

$$
\left.c(z, t)\right|_{t=0}=f_{3}(z), z_{a} \leq z \leq h
$$

in which: $z_{a}$ is the reference level and $f_{3}(z)$ is a given function from measured data. - The boundary condition at $z=h$ :

$$
\varepsilon_{s} \frac{\partial c}{\partial z}=-w_{s} c, z=h
$$

- The boundary condition at $z=z_{a}$ :

$$
\left.c(z, t)\right|_{z=z_{a}}=c_{a}(t) \text {, at } z=z_{a}
$$




$$
\text { or } \quad \varepsilon_{s} \frac{\partial c}{\partial z}=-w_{s} c_{a}, \text { at } z=z_{a}
$$

in which $c_{a}$ is a given formula or a measured time series.

The applied $c_{a}$ expression reads as:

$$
c_{a}=m \rho_{s} \frac{\left(\theta^{\prime}-\theta_{c r}^{\prime}\right)^{1.5}}{D^{*}},
$$

in which $\theta^{\prime}$ is instantaneous dimensionless bed shear stress related to the grains; $\theta_{c r}^{\prime}$ critical dimensionless bed shear stress known as Shields parameter; $\rho_{s}$ density of sediment; $D^{*}$ dimensionless particle diameter; and $m$ is an empirical coefficient (calibration) related to ripple characteristics $r, \lambda$, being ripple height and length respectively. The reference level is assumed to be equal to the effective bed roughness $\mathrm{k}_{\mathrm{s}}$, which is taken equal to the ripple height.

\section{Calibration of the model and numerical solutions}

The finite difference method based on implicit scheme of Crank-Nicolson was used to solve equations (3),(6)-(8) for horizontal velocity and the implicit upwind scheme was used in equations (9),(14)-(16) for vertical distribution of sediment concentration. It should be noted that the grids for flow and sediment diffusion equations are different and that a staggered grid has been used for more accurate and stable computation results.

Basic input values of the model are given in Table 2: $\mathrm{d}_{50}$ of the bed, $\mathrm{w}_{\mathrm{s}}=$ constant fall velocity of suspended sand (based on settling tests using suspended samples) and effective bed roughness $\mathrm{k}_{\mathrm{s}}$, which is taken equal to the measured ripple height.

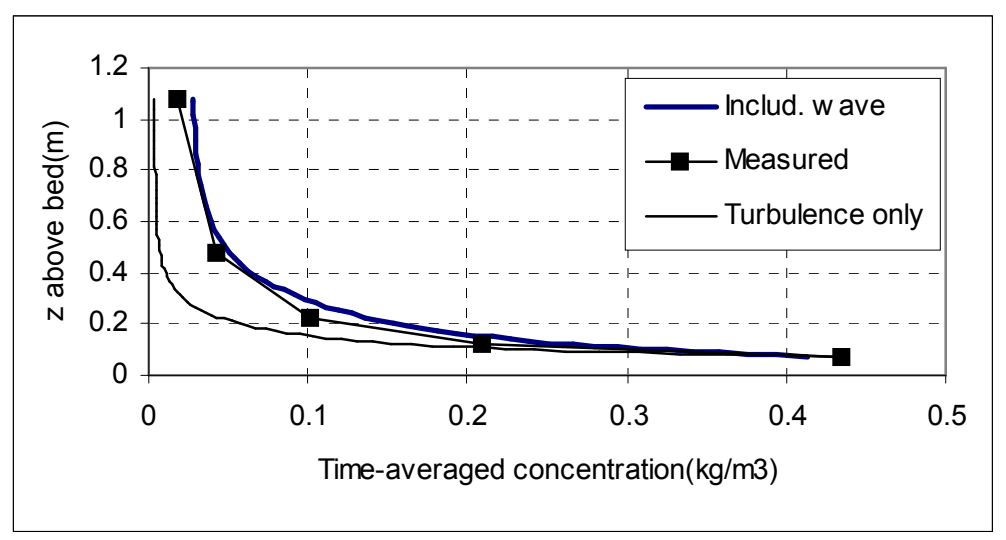

Fig. 3 Time-averaged concentration profile with and without wave-related diffusion (test G1)

First, the calibration of the vortex-related mixing coefficient is considered by fitting of measured and computed time-averaged sand concentrations. It is shown in Figure 3 that the time-averaged sand concentration is much too small when the turbulencerelated mixing coefficient is used only. Introducing a vortex-related mixing coefficient as determined by eq.(12), effectively improved the vertical distribution of sediment concentration considerably compared to the measurement results. Similar 
results were obtained for all other tests. Under the action of bottom-induced turbulent mixing the suspended sediment concentration mainly assembles near the bed and decreases quickly in the upward direction. All available test results (24) of timeaveraged sand concentrations have been used to determine the n-coefficient of the vortex-related mixing coefficient and the m-coefficient of the reference concentration expression by fitting of measured and computed time-averaged concentrations The results are given in Table 2.

Table 2. Input parameters for computations

\begin{tabular}{|c|c|c|c|c|c|c|c|c|c|}
\hline Test & $\begin{array}{l}\text { Max. } \\
\text { time for } \\
\text { computa } \\
\text {-tion } \\
\text { (s) }\end{array}$ & $\begin{array}{l}\text { Time-ave. } \\
\text { Velocity } \\
\text { at } z=h \mathrm{U}_{\mathrm{m}} \\
(\mathrm{m} / \mathrm{s})\end{array}$ & $\begin{array}{c}\text { Settling } \\
\text { velocity } \\
w_{s} \\
(\mathrm{~m} / \mathrm{s})\end{array}$ & $\begin{array}{c}\text { Bed } \\
\text { rough- } \\
\text { ness } \\
k_{s} \\
(\mathrm{~m})\end{array}$ & $\begin{array}{c}\text { Refere. } \\
\text { Level } \\
z_{\mathrm{a}} \\
\\
(\mathrm{m})\end{array}$ & $\begin{array}{c}\text { Coeff. } \\
\quad m \\
\text { in } \\
\text { Eq.(18) }\end{array}$ & $\begin{array}{c}\text { Exponent } \\
\text { Coeffici- } \\
\text { ent } \\
n \\
\text { in eq.(12) }\end{array}$ & $\begin{array}{l}\text { Ripple } \\
\text { height } \\
\quad \text { r } \\
\text { (m) }\end{array}$ & $\begin{array}{c}\text { Ripple } \\
\text { length } \\
\lambda \\
\text { (m) }\end{array}$ \\
\hline A1 & 892.0 & -0.03102 & 0.03 & 0.06 & 0.06 & 0.5 & 12.0 & 0.06 & 0.22 \\
\hline D1 & 897.0 & -0.00493 & 0.03 & 0.06 & 0.06 & 0.5 & 12.0 & 0.06 & 0.22 \\
\hline D3 & 888.5 & -0.00526 & 0.03 & 0.06 & 0.06 & 0.5 & 12.0 & 0.06 & 0.22 \\
\hline B1A & 890.5 & -0.03354 & 0.03 & 0.05 & 0.05 & 0.3 & 12.0 & 0.05 & 0.23 \\
\hline B2A & 893.5 & -0.03429 & 0.03 & 0.05 & 0.05 & 0.3 & 12.0 & 0.05 & 0.23 \\
\hline B1B & 117.5 & -0.03618 & 0.03 & 0.05 & 0.05 & 0.3 & 12.0 & 0.05 & 0.23 \\
\hline E3 & 894.5 & -0.01776 & 0.03 & 0.05 & 0.05 & 0.3 & 12.0 & 0.05 & 0.23 \\
\hline E4 & 652.5 & -0.01035 & 0.03 & 0.05 & 0.05 & 0.3 & 12.0 & 0.05 & 0.23 \\
\hline E5 & 896.5 & -0.00950 & 0.03 & 0.05 & 0.05 & 0.3 & 12.0 & 0.05 & 0.23 \\
\hline G1 & 892.0 & -0.03821 & 0.011 & 0.03 & 0.03 & 0.05 & 38.0 & 0.03 & 0.72 \\
\hline G2 & 894.5 & -0.01750 & 0.011 & 0.03 & 0.03 & 0.05 & 38.0 & 0.03 & 0.72 \\
\hline G4 & 892.5 & -0.02189 & 0.011 & 0.02 & 0.03 & 0.05 & 38.0 & 0.03 & 0.72 \\
\hline $\mathrm{J} 1$ & 897.0 & -0.03113 & 0.011 & 0.03 & 0.03 & 0.05 & 38.0 & 0.03 & 0.72 \\
\hline $\mathrm{J} 2$ & 895.5 & -0.03237 & 0.011 & 0.03 & 0.03 & 0.05 & 38.0 & 0.03 & 0.72 \\
\hline $\mathrm{J} 3$ & 356.0 & -0.03065 & 0.011 & 0.03 & 0.03 & 0.05 & 38.0 & 0.03 & 0.72 \\
\hline $\mathrm{H} 2$ & 823.5 & -0.03826 & 0.011 & 0.03 & 0.03 & 0.05 & 38.0 & 0.03 & 0.72 \\
\hline H3 & 898.5 & -0.03666 & 0.011 & 0.03 & 0.03 & 0.05 & 38.0 & 0.03 & 0.72 \\
\hline $\mathrm{H} 4$ & 894.5 & -0.02584 & 0.011 & 0.03 & 0.03 & 0.05 & 38.0 & 0.03 & 0.72 \\
\hline H5 & 893.0 & -0.03370 & 0.011 & 0.03 & 0.03 & 0.05 & 38.0 & 0.03 & 0.72 \\
\hline K2 & 898.5 & -0.02989 & 0.011 & 0.03 & 0.03 & 0.05 & 38.0 & 0.03 & 0.72 \\
\hline K4 & 892.5 & -0.03552 & 0.011 & 0.03 & 0.03 & 0.05 & 38.0 & 0.03 & 0.72 \\
\hline M1 & 891.5 & -0.04407 & 0.011 & 0.02 & 0.02 & 0.05 & 38.0 & 0.02 & 0.72 \\
\hline M2 & 836.5 & -0.03225 & 0.011 & 0.02 & 0.02 & 0.05 & 38.0 & 0.02 & 0.72 \\
\hline M4 & 474.5 & -0.03092 & 0.011 & 0.02 & 0.02 & 0.05 & 38.0 & 0.02 & 0.72 \\
\hline
\end{tabular}

The $n$-coefficient was found to depend on the grain size of the bed material: $n=12$ for coarse sand of $0.33 \mathrm{~mm}$ and $\mathrm{n}=38$ for finer sand of $0.16 \mathrm{~mm}$. A smaller $\mathrm{n}$-value implies a stronger vortex-related mixing, which seems realistic because the ripples were most pronounced (steep vortex ripples with $\mathrm{r} / \lambda=0.27$ ) in the tests with a coarse sand bed of $0.33 \mathrm{~mm}$.

The m-coefficient was found to be related to the ripple steepness $(\mathrm{r} / \lambda)$ with $m$-values in the range of $m=0.04$ for $\mathrm{r} / \lambda=0.042$ to $m=0.27$ for $\mathrm{r} / \lambda=0.5$. The results can be represented by 


$$
m=8.4\left(\frac{r}{\lambda}\right)^{2}-0.7 \frac{r}{\lambda}+0.06
$$

The measured velocities at $\mathrm{z}=1.075 \mathrm{~m}$ are used as the upper boundary condition of the model and the sand concentrations at the lower boundary condition of $z=z_{a}=0.075$ $\mathrm{m}$ are taken from measured concentrations as well as from equation (18). Based on these conditions, the model computations show quite good results for concentration at the lower elevations and for orbital velocity at the higher elevations (close to the boundaries). Further away from the boundaries the discrepancy between computed and measured values gradually increases. It also shows that the computed instantaneous results based on the measured reference concentration are much better than those from equation (18). Furthermore, the computed instantaneous concentration shows less strong oscillation than the measured concentration. This latter effect results in less accurate predictions of the wave-related sediment transport rate, as will be shown later. The vertical distribution of the time-averaged sand concentrations is not much affected by the type of boundary condition (measured data or equation 18).

For all the cases under consideration the computed instantaneous velocities outside the layer affected by the ripples (roughly two ripple heights above the mean bed; $\mathrm{z}>0.1$ to $0.2 \mathrm{~m}$ ) behave quite good in comparison to the measured velocities. Considerable errors occur close the bed in the vicinity of the ripples $(\mathrm{z}<0.1 \mathrm{~m})$.

Figure 4 shows computed and measured time-averaged velocity profiles for all 24 tests in terms of mean values (and standard error range) for each of the 5 data sets.
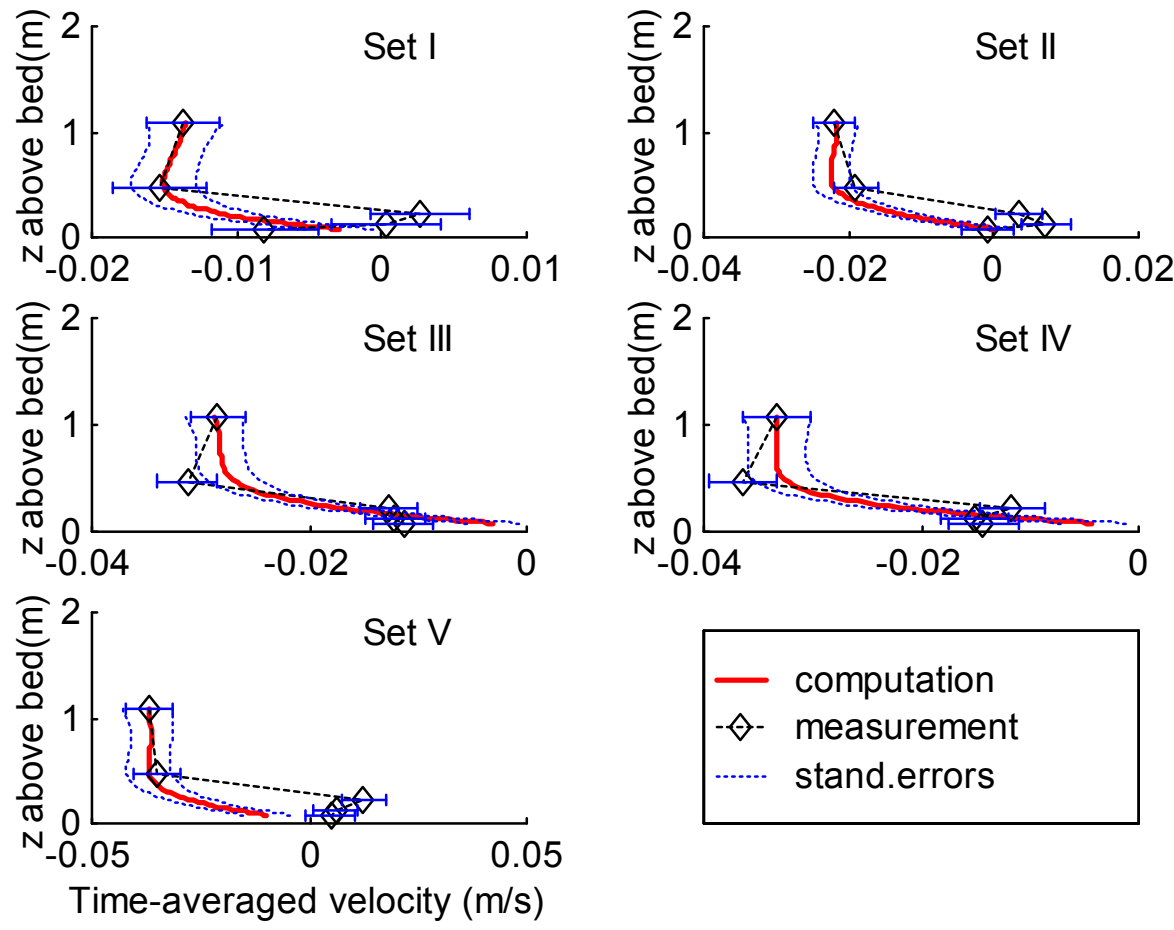

Fig. 4. Time-averaged velocities for combined tests

Figure 5 shows similar results for the time-averaged sand concentrations. The timeaveraged sand concentrations at the higher elevations $(\mathrm{z}>0.5 \mathrm{~m})$ are largest for data 
set $\mathrm{V}$ with the largest significant wave height and the finer sand of $0.16 \mathrm{~mm}$; whereas the near-bed concentrations are largest for the case with steep vortex ripples (data set I and II). These results show that a diffusion type model is quite good in simulating the time-averaged sand concentration distribution, provided that the vortex-related suspension mechanism is modelled by an effective mixing coefficient. The discrepancies of time-averaged concentration between using formula (18) and measured data for reference concentration are quite small. As regards the standard errors of both the computed and measured results, it shows that the velocities change significantly for each test due to the presence of the ripples, while the standard errors for concentration are more stable at the same levels.
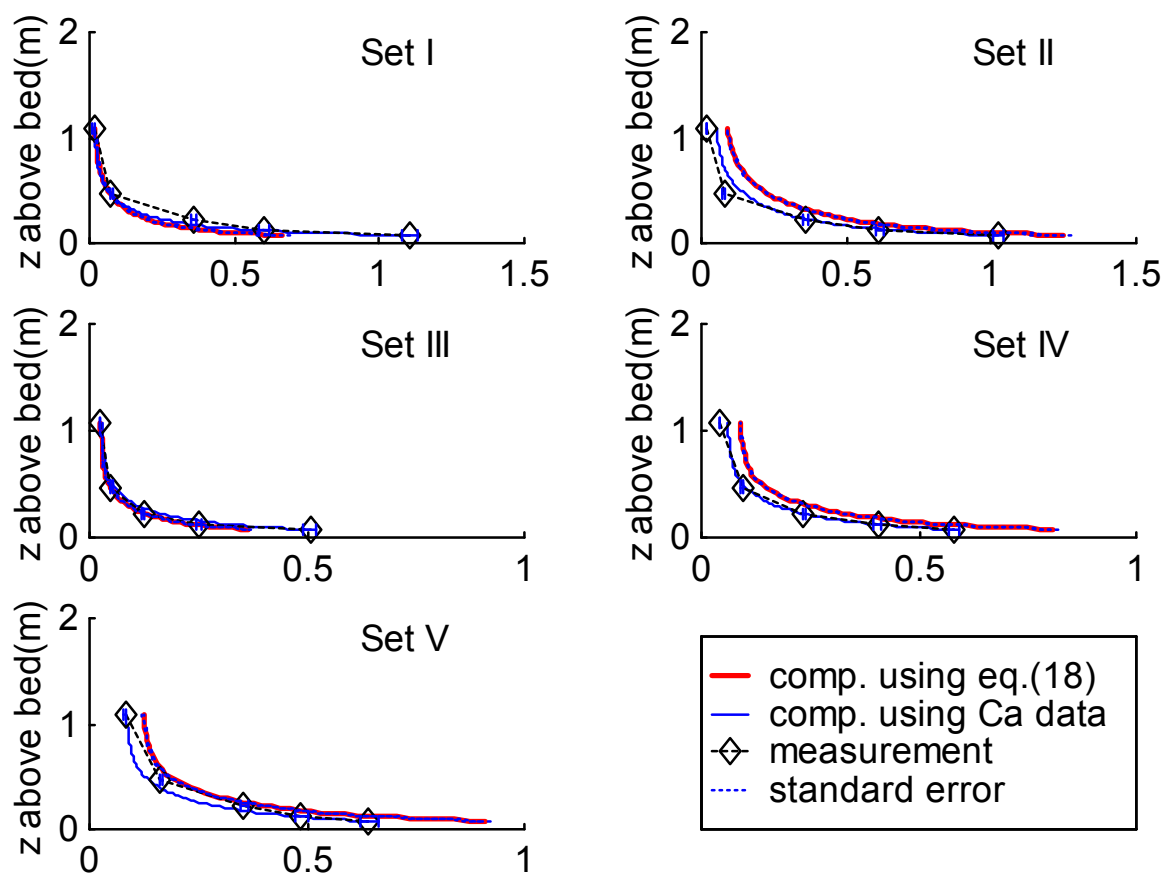

Time-averaged sand concentration

Fig. 5. Time-averaged sand concentrations for combined tests

\section{Evaluation of wave-related suspended transport rates}

The velocity and concentration computed by the model are the instantaneous quantities and they are represented as the sum of time-averaged component and the wave-related oscillations:

$$
\begin{aligned}
u(z, t) & =\bar{u}(z)+\widetilde{U}(z, t) \\
c(z, t) & =\bar{c}(z)+\widetilde{C}(z, t)
\end{aligned}
$$

The components due to high and low frequencies are not presented separately here. Therefore, the wave-related suspended sediment transport rate is defined as follows:

$$
Q_{w} \equiv(u-\bar{u})(c-\bar{c})=\overline{u \cdot c}-\bar{u} \cdot \bar{c}
$$

The model results in simulating the wave-related transport close to the bed are not accurate if the reference concentration is computed by a bed-shear stress related expression. The model results are considerably better if the measured sand 
concentrations of the lowest point are used as boundary condition for the fine sand bed of $0.16 \mathrm{~mm}$. This can also be observed from Figure 6 presenting computed depthintegrated transport rates (between the lowest $\mathrm{z}=0.075 \mathrm{~m}$ and the highest measurement point $\mathrm{z}=1.075 \mathrm{~m}$ ) versus measured depth-integrated transport rates (between the same points). The dashed lines are absolute error boundaries with values of $\pm 0.01 \mathrm{Kgm}^{-1} \mathrm{~s}^{-1}$.

There is a range of causes for the discrepancies between measured and computed wave-related suspended transport rates. As seen from the obtained results, the computed instantaneous concentrations do not agree well with the measured values although the time-averaged values are quite good. This problem is related to the geometry of the ripples as well as to the dynamics of entrainment and transportation of sediment particles from the rippled bed into the flow. It appears that the instantaneous behaviour of the vortices and associated sand concentrations can not be represented sufficiently accurate by a diffusion type model. Moreover, the interaction between sediment and fluid particles is not considered in the present model. The changes of fluid density and turbulence due to the presence of high near-bed sand concentrations may influence the dynamics process of flow and sediment.
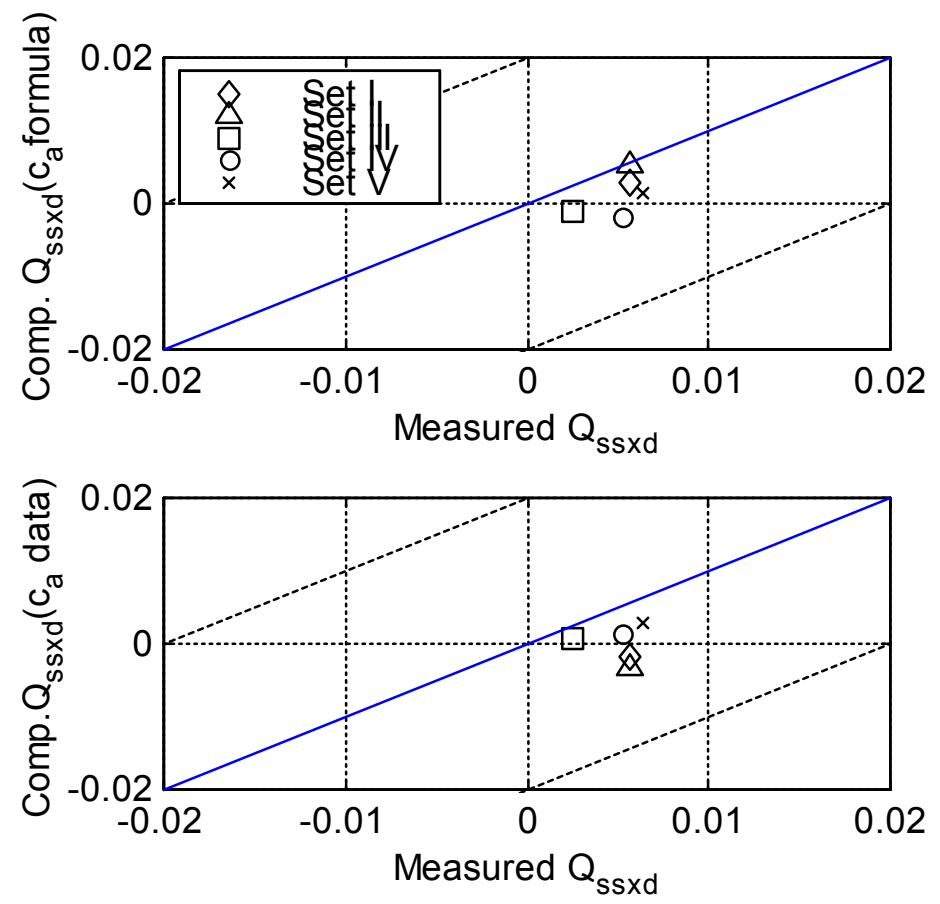

Fig. 6. Computed versus measured (depth-integrated) wave-related transport rates

\section{Discussion and conclusions}

The experimental results show that the ripple geometry is strongly affected by the bed material characteristics: steep vortex ripples with $r / \lambda=0.22$ to 0.27 for the $0.33 \mathrm{~mm}$ sand (data sets I and II) and relatively flat ripples with $\mathrm{r} / \lambda=0.04$ for $0.16 \mathrm{~mm}$ sand (data sets III, IV and V). The ripple steepness is a very important factor, because it decides the scale of bed roughness (see Hitching and Lewis, 1999) and hence has a strong influence on vertical distribution of horizontal flow velocity and sand concentration. As the precise measurement location with respect to the ripple crest is unknown for these Delta flume experiments, many repetitive measurements have been 
made to ensure representative sampling at various locations along the migrating ripples. Thus, the measured mean profiles of Figure 4 represent to some extent a spatially-averaged (over a ripple length) mean velocity profile. The error ranges give an indication of the horizontal variability involved. Comparison of measured and computed fluid velocities for the $0.33 \mathrm{~mm}$ sand (Figure 4) shows that a simple 1DVmodel is not very good for the values of the mean currents very close to steep ripples. Somewhat better results are obtained for data sets II and IV of the $0.16 \mathrm{~mm}$ sand bed with flat ripples using an effective bed roughness equal to the ripple height. Accurate simulation of the flow field near a rippled bed basically requires the application of a 2DV model in combination with a higher order turbulence closure ( $K-\varepsilon$ method) or direct large- scale eddy simulation (LES method), which is beyond the scope of the present study.

The time-averaged sand concentrations can be reasonably well simulated for both sand sizes using the classical diffusion approach, provided that the reference concentration near the bed is modelled with sufficient accuracy and that the vortexrelated mixing characteristics are taken into account (calibration). Neglecting the vortex-related mixing coefficient, yields time-averaged sand concentrations that are much too small (Figure 3). Further research is necessary to relate the reference concentration and the vortex-related mixing coefficient to relevant hydrodynamic, sediment and bed form parameters.

The measured instantaneous sand concentrations can not be simulated very accurately using a relatively simple reference concentration based on instantaneous bed-shear stress only; the effect of the ripple geometry has to be included to represent all sand concentrations peaks near the bed. The computed instantaneous sand concentrations at various elevations away from the bed show better agreement when measured data are used as bed boundary condition. For all cases the computed instantaneous sand concentrations show less strong oscillations than the measured data, which is an indication that the diffusion approach is not fully capable of simulating the instantaneous processes. However, this does not seem to affect the accuracy of the time-averaged sand concentrations and hence the current-related transport very much, but it is of major importance for the wave-related suspended transport processes. This latter process can not be simulated very accurately by the present 1DV model in case of a rippled bed, especially when the reference concentration is not accurately modelled.

For all cases the measured wave-related suspended transport rates due to the high frequency waves are directed onshore, whereas the current-related suspended transport rates are directed offshore. The transport rates due to low frequency are of minor importance and have a tendency for offshore direction similar to time-averaged transport components.

The results of the present study for sand transport under irregular waves over a bed of $0.16 \mathrm{~mm}$ sand and $0.33 \mathrm{~mm}$ sand can be summarized by the following conclusions:

- The ripple geometry is strongly affected by the bed material characteristics: steep vortex ripples with $\mathrm{r} / \lambda=0.22$ to 0.27 for the $0.33 \mathrm{~mm}$ sand (data sets I and II) and relatively flat ripples with $\mathrm{r} / \lambda=0.04$ for $0.16 \mathrm{~mm}$ sand (data sets III, IV and V);

- The suspended sediment transport mainly occurs in the near-bed layer with thickness of about 0.3 to $0.5 \mathrm{~m}$ (water depth of $4.55 \mathrm{~m}$ ), which is roughly equivalent to 10 to 20 times the ripple height;

- The measured wave-related suspended transport due to the high frequency waves is directed onshore, whereas the current-related suspended transport is directed 
offshore; the transport rates due to low frequency are of minor importance and have a tendency for offshore direction similar to time-averaged transport components;

- The onshore-directed wave-related suspended transport increases with wave height $\left(\mathrm{H}_{1 / 3}\right.$ between 1 and $\left.1.5 \mathrm{~m}\right)$ and decreases with sand size; the latter effect is related to the effect of decreasing ripple steepness and hence less strong vortex motions for decreasing sand size;

- The 1DV model based on the classical diffusion approach shows good ability to simulate the time-averaged suspended sand concentrations and hence the currentrelated suspended transport in the ripple regime, provided that the reference concentration near the bed and the vortex-related mixing are represented with sufficient accuracy; both parameters are strongly related to the ripple characteristics.

\section{ACKNOWLEDGEMENTS}

The experiments were done in the Delta flume of Delft Hydraulics under the framework of the LIP-program of the European Commission. The Proudman Oceanographic Laboratory is gratefully acknowledged for giving the opportunity to the Dep. of Phys. Geography of the Univ. of Utrecht to participate in the experiments. The first author would like to express his gratitude to the University of Utrecht for providing the scholarship to carry out the present research. The SEDMOC project sponsored (under contract MAS-3-CT97-0015) by the European Commission Directorate General XII for Science, Research and Development is gratefully acknowledged for providing funds to support the analysis of the data set.

\section{REFERENCES}

Bakker,W.T., 1974. Sand concentration in oscillatory. Proc. $14^{\text {th }}$ ICCE, Copenhagen, ASCE,Vol.2, pp.1129-1148

Brevik, I., 1981. Oscillatory rough turbulent boundary layers. ASCE WW3, No.107, pp.175-188.

Dang Huu,Ch. and Grasmeijer, B.T., 1999. Analysis of sand transport under regular and irregular waves in large-scale wave flume. Report R99-05, IMAU, Utrecht University, The Netherlands.

Fredsoe, J., Andersen, O.H. and Silberg, S., 1985. Distribution of suspended sediment in large waves. Journal of Waterway, Port, Coastal and Ocean Engineering, Vol.111, No.6, pp.1041-1059

Grasmeijer, B. and Van Rijn, L.C., 1999. Transport of fine sands by currents and waves III: Breaking waves over barred profile with ripples. Journal of Waterway, Port, Coastal and ocean Engineering, Vol. 125, No. 2, pp. 71-79

Hitching, E. and Lewis, A.W., 1999. Bed roughness over vortex ripples. Proc. $4^{\text {th }}$ Int. Symp. on Coastal Engineering and Coastal Sediment Processes, Long Island, New York, June 21-23, 1999, ASCE, Vol.1, pp.18-30

Horikawa, K., Watanabe, A., 1968. Laboratory study on oscillatory boundary layer flow. Proc. $11^{\text {th }}$ Coastal Eng. Conf., pp.467-486.

Jonsson, I.G., 1963. Measurements in the turbulent wave boundary layer. Proc. $10^{\text {th }}$ Congress IAHR, pp.85-92.

Kajiura, K., 1968. A model of the bottom boundary layer in water waves. Bull. Earthquake Res. Inst, Vol.46, pp.75-123. 
Nielsen,P., 1992. Coastal bottom boundary layers and sediment transport. World Scientific Publishing Corporation, New York

Osborne, P.D. and Greenwood, B.G., 1992. Frequency-dependent cross-shore suspended sediment transport 2: A barred shoreface. Marine geology 106, pp. 25-51

Osborne, P.D. and Vincent, C.E., 1996. Vertical and horizontal structure in suspended sand concentrations and wave-induced fluxes over bed forms. Marine Geology 131, pp. $195-208$

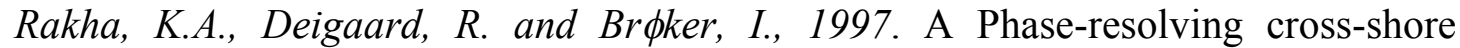
sediment transport model for beach profile evolution. Coastal Eng. 31, p. 231-261

Ribberink, J.S., 1998. Bed-load transport for steady flows and unsteady oscillatory flows. Coastal Engineering 34, pp. 59-82

Ribberink, J.S. and Al-salem, A.,1991. Near-bed sediment transport and suspended sediment concentrations under waves. Proc. Int. Symposium on: "The transport of suspended sediment and its mathematical modelling", Florence, 1991

Ribberink, J.S. and Al-salem, A.,1995. Sheet flow and suspension of sand in oscillatory boundary layers. Coastal Engineering 25, pp.205-225

Van Rijn, L.C. 1993. Principles of sediment transport in rivers, estuaries and coastal seas, Aqua Publications, Amsterdam, The Netherlands, p. 730

Van Rijn, L.C., 1998. Principles of coastal morphology. Aqua Publications, Amsterdam, The Netherlands, p. 750

Van Rijn, L.C. et al., 1993. Transport of fine sands by currents and waves I. Journal of Waterway, Port, Coastal and ocean Engineering, Vol. 119, No. 2, pp. 123-143

Van Rijn, L.C. and Havinga, F.J., 1995. Transport of fine sands by currents and waves II. Journal of Waterway, Port, Coastal and ocean Engineering, Vol. 121 No. 2, pp. $123-133$ 